\title{
Life Cycle Planning of Battery Energy Storage System in Off-grid Wind-Solar-Diesel Microgrid
}

\author{
Yuhan Zhang ${ }^{1,2}$, Jianxue Wang ${ }^{1 *}$, Alberto Berizzi ${ }^{3}$, Xiaoyu Cao ${ }^{1}$ \\ ${ }^{1}$ School of Electrical Engineering, Xi'an Jiaotong University, Xi'an, China \\ ${ }^{2}$ State Grid Shaanxi Electric Power Company Economic Research Institute, Xi'an, China \\ ${ }^{3}$ Energy Department, Politechnico di Milano, Milan, Italy

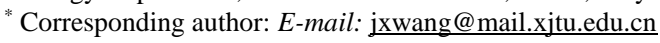

\begin{abstract}
For off-grid microgrids in remote areas (e.g., sea islands), proper configuring the battery energy storage system (BESS) is of great significance to enhance the power-supply reliability and operational feasibility. This paper presents a life cycle planning methodology for BESS in microgrids, where the dynamic factors such as demand growth, battery capacity fading, and components' contingencies are modelled under a multi-timescale decision framework. Under a yearly timescale, the optimal DER capacity allocation is carried out to meet the demand growth, while the investment decisions of BESS are made periodically to yield the optimal sizing, type selection, and replacement plans of BESS during the entire lifetime of microgrid. Then, under an hourly time-scale, the long-term probabilistic sequential simulation is adopted to comprehensively evaluate the investment decisions and derive detailed operation indicators. Moreover, a decompositioncoordination algorithm is developed to address the presented planning model, which iteratively strengthens the feasible space of investment decision model by substituting the operation indicators until an acceptable sub-optimal solution is obtained. Case studies on a wind-solar-diesel microgrid in Kythnos Island, Greece, illustrate the effectiveness of the proposed method. This study provides a practical and meaningful reference for BESS planning in off-grid microgrids.
\end{abstract}

\section{Introduction}

In remote areas (e.g., high mountains, sea islands), the fuel supply (e.g., coal and natural gas) is costly. Also, it could not be economical to expand the utility distribution network to energize the remote users. On the other hand, these regions typically possess abundant natural resources, which proliferates the application of off-grid microgrids with hybrid renewable energy and flexible loads as a clean and sustainable alternative of power supply [1]-[2].

In these off-grid microgrids, battery energy storage system (BESS) is essential to cope with the supply-demand mismatches caused by the intermittent and volatile nature of renewable energy generation [3]. However, the functionality of BESS in off-grid microgrids requires it to bear the large charge/discharge power, deep cycling and frequent charging process, which may lead to nonnegligible and irreversibly degradation of storage capacities. Because of the capacity degradation, the energy storage modules in microgrids will be replaced for several times. In addition to the capital investment, the expense of facility replacement is also a crucial factor in the economic measures of microgrid planning. Hence, the optimization of BESS investment and replacement decisions should be fully considered at the planning stage of off-grid microgrids [4].

Generally, the main task of BESS planning contains the optimal sizing and type selection of storage modules, which has been extensively studied in current literatures through either optimization methods [5] or commercial software, e.g., Hybrid Optimization Model for Electric Renewable software (HOMER) [6]. Paper [7]-[8] presented the design approaches of hybrid electrical energy storage (HEES), where the power processed by HEES was separated into the low frequency and the high frequency parts. In the proposed methods, the low frequency part was leveled by energy storage batteries while the high frequency part was compensated by the quick-response power storage devices. Paper [9] adopted the wavelet analysis to make the investment decision of hybrid energy storage system. Paper [10] applied the discrete Fourier transform (DFT) method to coordinate the sizing of BESS and diesel generators. Note that in a practical microgrid, the operation of BESS is highly correlated to the power generation of other distributed energy resources (DER), e.g., wind turbines, solar panels, and diesel generators. Thus, it is essential to coordinate the optimal configuring of BESS and other DERs (as generation sources), which could be hardly captured by the modeling in the aforementioned studies and require for more advanced optimization tools [11]. Moreover, the component sizing has a tight relation with many factors, e.g., long-term trend of load demand, precise component modeling, different energy management strategies and component contingencies. For example, paper [12] shows the importance of utilizing accurate battery models in sizing standalone photovoltaic systems. Paper [13] puts emphasis on the positive effect of demand response programming in component sizing.

We notice that the joint planning of BESS and DERs, which is an effective way to improve the entire performance of microgrids, has been discussed in some current studies. Paper [14] presented a two-layer co-optimization framework for the sizing of different energy resources in a hybrid renewable energy microgrid. In this study, particle swarm optimization (PSO) method was adopted to generate and sort the planning alternatives following a cost-minimization criterion, while the planning schemes were assessed by an analytical approach. Paper [15] designed a heuristic sizing strategy for a wind-solar-battery microgrid based on several principle, e.g., high reliability, cost-minimization, and the complementary of natural resource. Paper [16] implemented the sequential Monte Carlo simulation (MCS) under a pattern search (PS) optimization framework to seek for a least-cost sizing plan of a microgrid with BESS integration. 
As mentioned in [14]-[16], the combination of heuristic optimization framework (e.g., PSO, genetic algorithm (GA), and ant colony algorithm) and performance assessment methods provides a generic computational framework for BESS planning problems. The performance assessment criteria could be defined as a variety of reliability and economic indicators, e.g., loss of power supply probability (LPSP), net present cost (NPC), and levelized cost of energy (LCE) [17]. To capture the coherent and stochastic behaviors (incurred by intermittent renewable energy generation, load variation, and unexpected components' failure, etc.) of microgrids operation, the probabilistic sequential simulation methods are preferable for performance assessment. Paper [18] proposed a two-step sequential MCS method along with the modeling of battery packs to evaluate the reliability and economics of a microgrid considering the random outputs of renewable energy sources. The evaluation results can be used to compare and sort the planning alternatives. However, the impact of operation problems was only simply modeled in the aforementioned sizing methods. Paper [19] proposed a generation scheduling based approach to optimize the sizing of BESS.

Based on our previous studies in [20], the operational feasibility of BESS integrated microgrids could be largely influenced by dynamic and random factors under different timescales. The short-term random factors (also known as random uncertainties), e.g., renewable energy generation and component contingencies, have been maturely modeled or simulated in the current literatures [16], [18]. Nevertheless, another category named long-term dynamic factors, e.g., load growth and the capacity fading of energy storage modules, are not well considered in the previous studies of BESS planning. Compared to the grid-connected systems, the off-grid microgrid cannot receive the power and reserve supports from the external utility grid, which makes it more vulnerable to the operational risks introduced by these dynamic factors. In this regard, proper planning strategies should be put forward to trace the long-term varying trend of key design factors such as load growth and storage capacity degradation, in the BESS planning for an off-grid microgrid, which motivates our research in this paper.

This paper studied the life cycle planning of BESS considering battery performance degradation, load growth and component random contingencies in an off-grid windsolar-diesel microgrid. Compared to the current literatures, our major contributions can be summarized as:

1) We propose a multi-stage and multi-timescale BESS planning formulation to integrally consider the longterm dynamic and short-term random factors. On one hand, the strategies of storage capacity expansion and replacement are developed under a yearly timescale to handle the long-term dynamic changes of demand capacity and available storage capacity. On the other hand, the hourly operation of microgrids (with BESS and DERs) is elaborately modeled to capture the short-term randomness of component contingencies as well as renewable energy generation.

2) To address the computational challenge of the proposed formulation, we apply the decompositioncoordination algorithm [21], [22] to BESS planning, where the complex mixed-integer program problem is separated into two simpler parts. The first part is to optimize the capacity allocation of DERs and the multi-period sizing plan of BESS. Then, in our second part, the probabilistic sequential long-term simulation is carried out based on the operational modeling of off-grid microgrids. Coordination variables generated by simulation will be fed back to strengthen the solution space of the first subproblem iteratively until a specified convergence precision is met. In addition, case studies are conducted to validate the developed solution algorithm.

The rest of the paper is presented as follows: Section 2 establishes the multi-timescale modeling of BESS. Section 3 puts forward the overall framework of research and the life cycle planning model of BESS. Then decompositioncoordination algorithm is introduced in Section 4. Section 5 presents the case studies. The conclusions are in Section 6.

\section{Multi-timescale modeling of BESS}

An off-grid wind-solar-diesel microgrid is studied in this paper. The configuration of mentioned microgrid and the basic models of its components are shown as Fig.1. All DC-based renewable energy sources and energy storage units are connected to a DC bus to facilitate the control of distributed power. The controllable diesel generator and AC load are connected to $\mathrm{AC}$ bus, thus reducing the probability of power outages. The AC/DC rectifier or DC/DC converter is simplified as a coefficient of $95 \%$ as the efficiency of power output of each component. It combines the strengths of different energy sources to achieve wind-solar complementary, flexible operation and high efficiency.

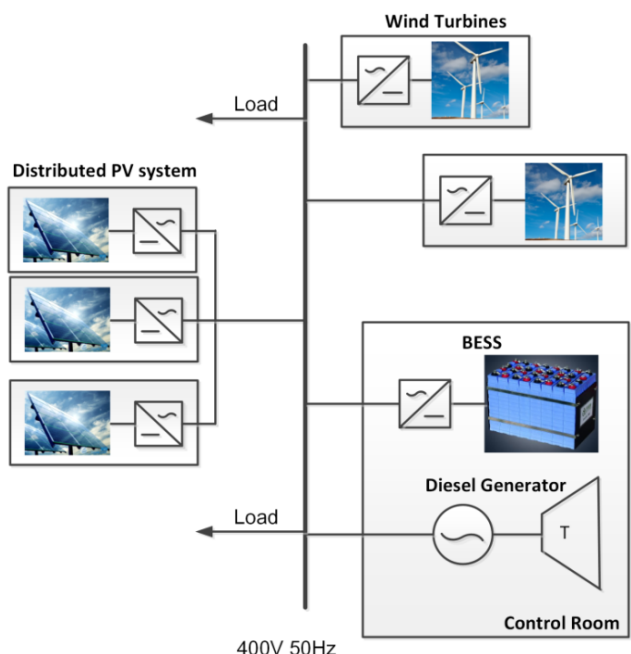

Fig.1. Configuration of an off-grid wind-solar-diesel microgrid 
For off-grid microgrids in remote areas and islands, battery energy storage system (BESS) is of great importance for power supply reliability and power balance. However, BESS usually faces severe variable charging condition battery capacity degradation cannot be neglected in practical use, especially along the life cycle of microgrid. In order to fully describe the behaviour of BESS, multi-timescale modeling is proposed in this paper. In the short-term, BESS charge/discharge control is considered in the operation. While in the long run, BESS capacity degradation has to be modelled to illustrate the realistic battery state.

\subsection{Charge/discharge control of BESS}

The charging or discharging state of battery storage system is determined by the matching condition of renewable energy resources and load demand. The power difference between the power output of WT, PV and the load demand can be calculated as follows:

$P_{\text {diff }}(t)=\eta_{\text {inv }}\left[P_{p v}(t)+P_{w t}(t)\right]-P_{L}(t)$

where $P_{w t}(t), P_{p v}(t)$ and $P_{L}(t)$ are power generation of WT, PV and the load demand in hour $t$, respectively. $\eta_{\text {inv }}$ is bidirectional bus converter efficiency.

1) If the output power of WT and PV exceeds the load demand, the batteries will be charged. The charge power in hour $t P_{c}(t)$ is determined as follows:

$P_{c}(t)= \begin{cases}\eta_{c} \eta_{\text {inv }} P_{\text {diff }}(t) & \text { for } P_{\text {diff }}(t)<P_{\text {ch,max }} \\ \eta_{c} \eta_{\text {inv }} P_{c h, \text { max }} & \text { for } P_{\text {diff }}(t)>P_{c h, \text { max }}\end{cases}$

where here $P_{c h, \text { max }}$ is the maximum charge power BESS allowed. $\eta_{c}$ is the charging efficiency.

After the charging process, the state of charge (SOC) will change accordingly. The SOC in hour $t$ can be obtained as:

$\operatorname{SOC}(t)=\operatorname{SOC}(t-1)+P_{c}(t) \cdot \Delta t / Q_{b a t}$

where $Q_{b a t}$ is the rated capacity of BESS (kWh).

2) When the output power of renewable energy resources cannot meet the load demand, the batteries will get discharged. The discharge power in hour $t P_{\text {dis }}(t)$ is determined as follows:

$P_{\text {dis }}(t)= \begin{cases}\frac{-P_{\text {diff } f}(t)}{\eta_{\text {dis }} \eta_{\text {inv }}} & \text { for } P_{\text {dis,max }}>-P_{\text {diff }}(t) \\ \frac{P_{\text {dis }} \text { max }}{\eta_{\text {dis }} \eta_{i n v}} & \text { for } P_{\text {dis,max }}<-P_{\text {diff }}(t)\end{cases}$

where $\eta_{d i s}$ is discharge efficiency, $P_{d i s, \max }$ is the maximum discharge power. The SOC in hour $t$ therefore changed into:

$\operatorname{SOC}(t)=\operatorname{SOC}(t-1)-P_{\text {dis }}(t) \Delta t / Q_{b a t, y}$

3) When the load demand is exactly balanced by renewable power output, the batteries will neither be charged nor be discharged.

\subsection{Capacity degradation modeling of BESS}

Battery testing shown in paper [23] demonstrates that the battery capacity is linearly declining along with the increment of cycles. And in the variable charging condition, the capacity of BESS decreases faster than that is in the constant charging condition. The larger the depth of discharge is, the faster the battery capacity fades.

Assuming that charge/discharge process of BESS in one year are recorded, the annual energy absorbed by BESS can be cumulated as $Q_{y}$. The number of full cycles in the $y$ th year $C_{y}$ can be calculated as below:

$C_{y}=\frac{Q_{y}}{P_{d i s, \max }}$

The standard criterion for battery failure is that the capacity of battery degrades to $80 \%$ of its rated capacity. The corresponding number of cycles one battery can run till it fails is called cycles to failure $C T F_{\text {end }}$. To simplify the characteristic of battery capacity loss process, the relationship of remaining available capacity and number of full cycles can be linearized [24] and expressed as follows:

$Q_{b a t, y+1}=Q_{b a t, y}-\frac{C_{y}\left(Q_{i n i}-Q_{e n d}\right)}{C T F_{\text {end }}}$

where $Q_{b a t, y}$ is the remaining available capacity in the end of the $y$ th year. $Q_{i n i}$ is the rated capacity. $Q_{e n d}$ is the end capacity at the last year of its minimum lifetime, namely $80 \%$ of the rated capacity $Q_{\text {ini }}$.

\section{Life cycle planning of battery energy storage system}

As mentioned before, the planning of BESS is in conjunction with the optimal capacity configuration of DERs. The planning of DERs and BESS should be implemented at the same time. A multi-stage planning framework is established for the life cycle planning of BESS in off-grid wind-solar-diesel microgrid. The framework is decomposed into three stages and depicted in Fig.2.

In the first stage, microgrid system structure and equipment type are determined. Scenario database renewable energy is generated based on localized information. The life-cycle capacities of DERs are preliminarily allocated in this stage.

In the second stage, the investment-decision model is established to derive the type and size of BESS. Long-term simulation is adopted to verify the feasibility of the scheme and obtain practical operation variables. As shown in Fig.2, these practical operation variables will be fed back to the investment-decision model as coefficients in a new iteration. The iterative approach keeps running until the variation of the operation variables is limited within a pre-defined range.

In the third stage, economic, reliability and operation performance indicators of the microgrid are evaluated. If the performance of sizing result is not satisfied, constraints will be adjusted and the process will be restarted. 


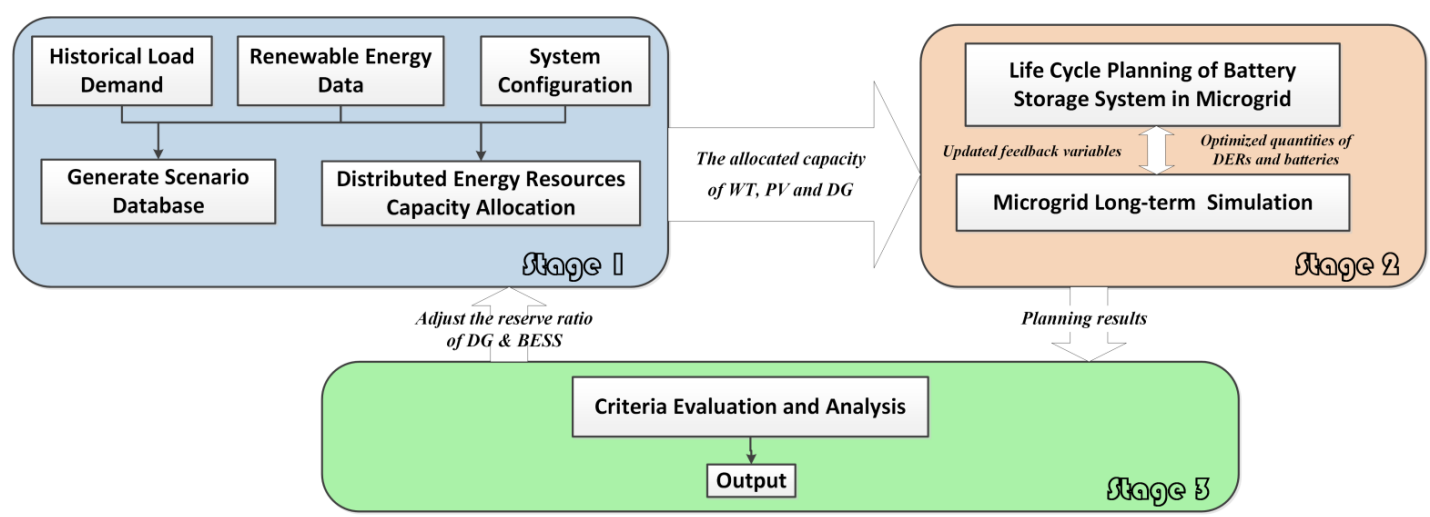

Fig.2. The framework of life cycle planning of battery energy storage system

\subsection{Scenario database generation}

Known the location of the microgrid, the monthly mean value of wind speed and irradiation can be estimated via NASA Surface meteorology and Solar Energy service [24].

The hourly wind speed for one year can be generated based on monthly wind speed and be directly adopted in Monte Carlo iterations [25]. The hourly output power of wind turbine (WT) can be estimated as follows:

$p_{w t}(t)=\left\{\begin{array}{lr}0 & v \leq v_{c i} \text { and } v \geq v_{c o} \\ p_{w t, n} \frac{v(t)^{3}-v_{c i}^{3}}{v_{n}^{3}-v_{c i}^{3}} & v_{c i} \leq v \leq v_{n} \\ p_{w t, n} & v_{n} \leq v \leq v_{c o}\end{array}\right.$

where $p_{w t, n}$ is the nominal output power of wind turbine. $v_{c i}$ is the cut-in wind speed. $v_{c o}$ is the cut-out wind speed and $v_{n}$ is the rated wind speed of WT.

Paper [26] provides a methodology on the basis of monthly radiation to generate hourly solar radiation scenarios for one year. The output power of a photovoltaic module is mainly determined by two factors: ambient temperature and solar radiation. Several solar radiation scenarios can be adopted for Monte Carlo simulation. According to module characteristic, the output power of a photovoltaic cell (PV) can be obtained as follows:

$p_{p v}(t)=P_{S T C} G_{C}(t)\left[1+k\left(T_{C}(t)-T_{S T C}\right)\right] / G_{S T C}$

where $G_{S T C}, P_{S T C}, T_{S T C}$ are the PV cell solar irradiance, maximum power output and the temperature under standard condition, respectively. $G_{C}$ and $T_{C}$ are the solar radiation and the surrounding temperature in hour $t$ respectively. $k$ is the temperature coefficient.

Therefore, hourly renewable power output scenario database is generated in advance. The row vector of hourly power output of a wind turbine and a photovoltaic module for one year is demonstrated as $\boldsymbol{p}_{w t}$ and $\boldsymbol{p}_{p v}$ respectively.

\subsection{Capacity allocation of DERs}

Distributed energy resources (DERs) are power generation units located within the electric distribution grid. They can be divided into intermittent resources, such as wind turbine, photovoltaic array, and controllable resources like diesel generator. The life-cycle sizing of DERs is optimized year by year. For the $y$ th year, the number of wind turbine, photovoltaic module and diesel generator $N_{w t}^{y}$ $N_{p v}^{y}$ and $N_{d g}^{y}$ is solved.

Renewable energy resources are chosen preferentially as the energy suppliers of the microgrid. The balanced load demand can be defined as the difference between load demand and output power of WT and PV. The row vector of hourly balanced load demand $\boldsymbol{P}_{\text {net }}^{y}$ can be calculated as:

$\boldsymbol{P}_{\text {net }}^{y}=\boldsymbol{P}_{L}(1+\alpha)^{\mathrm{y}-1}-N_{w t}^{y} \boldsymbol{p}_{w \boldsymbol{t}}-N_{p v}^{y} \boldsymbol{p}_{\boldsymbol{p} v}$ where $\boldsymbol{P}_{L}$ is the row vector of starting year microgrid hourly load demand and $\alpha$ is the average load growth factor.

3.2.1 Capacity allocation of renewable DERs: The annual variation [15] is introduced to show how the renewable power output compensates the electrical load demand. For the $y$ th year, the optimization problem is to minimize the annual variation $D_{L}^{y}$ [15]. The number of WT and $\mathrm{PV}, N_{w t}^{y}$ and $N_{p v}^{y}$, can be allocated by solving this model for each year.

$\min D_{L}^{y}=\min \boldsymbol{P}_{n e t}^{y} \cdot\left(\boldsymbol{P}_{n e t}^{y}\right)^{\mathbf{T}}$

Related constraints are listed as below:

1) The numbers of WT and PV are confined by the area of the system.

$N_{w t}^{y} \leq\left(\frac{L}{6 d}+1\right)\left(\frac{W}{4 d}+1\right)$

$N_{p v}^{y} \leq \frac{s_{p v}}{s_{\text {panel }}}$

where $L$ and $W$ are the length and width of the wind farm; $d$ is the rotor diameter of WT; $S_{p v}$ is the total area where PV can be installed; $S_{\text {panel }}$ is the area of one PV panel.

2) From energy utilization point of view, extreme high penetrated renewable energy may not fully meet the load. On the contrary, it may lead to the instability of the 
system. In addition, diesel generator will have their annual electricity production no less than 0 . The constraint of net electricity demand is expressed as:

$W_{\text {net }}^{y}=W_{L}^{y}-\sum_{t=1}^{8760}\left(N_{p v}^{y} p_{p v}(t)+N_{w t}^{y} p_{w t}(t)\right) \geq 0$

where $W_{\text {net }}^{y}$ is the net electricity demand. $W_{L}^{y}$ is the total annual electricity consumption.

3.2.2 Capacity allocation of controllable DER: Diesel generator (DG) is a kind of controllable power generation equipment. By consuming diesel fuel, the diesel prime mover drives the generator to supply electricity. In this study, its real-time power output depends on the power balance of microgrid.

The balanced load demand $\boldsymbol{P}_{\text {net }}^{y}$, as discrete samples, can be decomposed into DC component, low-frequency and high-frequency power fluctuation component. FFT is adopted as a filter to separate these components [2]. For high-frequency power fluctuations $\boldsymbol{P}_{h f}^{y}$, BESS with rapid response time is used. While the DC component and lowfrequency parts that represent seasonal variation of the balanced load demand are covered by diesel generator.

The number of diesel generators could be achieved based on the determined capacities of WT and PV. In operation, some reserve power is maintained in case of accident or prediction errors. An operating reserve ratio $r_{d g}$ is introduced to cope with sudden power unbalance. $r_{d g}$ is defined as the ratio between extra reserve power and the maximum power demand. The number of diesel generators in the $y$ th year is determined as follows:

$N_{d g}^{y}=\left(1+r_{d g}\right) \frac{\max \left(\boldsymbol{P}_{n e t}^{y}-\boldsymbol{P}_{h f}^{y}\right)}{p_{d g, n}}$

where $p_{d g, n}$ is the rated capacity of a single DG.

\subsection{Investment-decision model of battery energy storage system}

The life cycle planning of BESS is on the basis of allocated capacities of DERs. The target of the model is to choose one type from alternative types of batteries and offer the optimal capacity for BESS along the project lifespan. In this study, the lifetime of each kind of battery is predetermined and the replacement is implemented group by group during the project lifespan. The storage batteries will be replaced and recycled at the beginning of a year.

The objective of investment-decision model is to minimize the life cycle cost of battery energy storage system. The total cost is comprised of investment and replacement $(I \& R)$ cost, operation and maintenance $(\mathrm{O} \& \mathrm{M})$ cost, and recovery cost.

$\min L C C=\min \left(\sum_{y=1}^{Y_{S}}\left(\frac{1}{1+r}\right)^{y-1}\left(C_{I R}^{y}+C_{O M}^{y}+C_{R C}^{y}\right)\right)$

where $r$ is the interest rate and the investment and replacement, operation and maintenance and recovery cost is listed as follows:

$C_{I R}^{y}=\sum_{i=S_{1}}^{S_{n}} C_{S_{i}}^{I R} N_{\text {new }, S_{i}}^{y}$

$C_{O M}^{y}=\sum_{i=S_{1}}^{S_{n}} C_{S_{i}}^{O M} N_{o p, S_{i}}^{y}$
$C_{R C}^{y}=\sum_{i=S_{1}}^{S_{n}} C_{S_{i}}^{R C} N_{r e, S_{i}}^{y}$

where $N_{\text {new }, S_{i}}^{y}, N_{o p, S_{i}}^{y}, N_{r e, S_{i}}^{y}$ are the numbers of batteries newly installed, the batteries in operation and the recycled batteries of $S_{i}$ type in the $y$ th year, respectively. $C_{S_{i}}^{I R}$ is the capital cost of one $S_{i}$ type battery unit (€/battery), $C_{S_{i}}^{O M}$ is the O\&M cost of one $S_{i}$ type battery unit (€/battery), $C_{R C}^{y}$ is the recycling cost of one $S_{i}$ type battery unit ( $€ /$ battery).

The objective function of BESS planning is subject to a series of constraints, which can be classified into uniqueness constraint, numerical relationship, power balance and energy balance.

1) Uniqueness constraint

In this paper, it is assumed that three kinds of batteries can be chosen. And the microgrid installs only one type of batteries as the energy storage device. $B_{S_{i}}^{y}$ is a $0-1$ variable to indicate whether type $S_{i}$ batteries will be chosen as the storage system. Therefore the sum of all the 0-1 variables representing battery type from $S_{1}$ to $S_{n}$ is equal to 1. It means if $B_{S_{1}}^{y}$ is 1 , the rest other $0-1$ variables are all equal to 0 so that the $S_{1}$ type battery is chosen to be installed $\sum_{i=S_{1}}^{S_{n}} B_{S_{i}}^{y}=1$

2) Numerical relationship of battery installation.

The number of batteries in operation in the $y$ th year is the difference of the total number of newly installed batteries and that of recycled batteries since the first year:

$N_{o p, S_{i}}^{y}=\sum_{n=1}^{y}\left(N_{n e w, S_{i}}^{n}-N_{r e, S_{i}}^{n}\right)$

And the installed batteries have to operate at least for a predetermined lifetime. It can be expressed as follows:

$N_{r e, S_{i}}^{y}=\left\{\begin{array}{cc}0 & y \leq Y_{S_{i}} \\ N_{\text {new }, S_{i}}^{y-Y_{S_{i}}} & Y_{S_{i}}<y \leq Y_{S}\end{array}\right.$

where $Y_{S_{i}}$ is the lifetime of the $S_{i}$ type batteries.

Unless the current group of batteries is worn out, no battery will be installed during the lifetime.

$N_{\text {new }, S_{i}}^{y}=0$,if $y \neq k Y_{S_{i}}+1, k=0,1,2,3 \ldots$

Assuming that all power sources fail simultaneously, the battery storage system ought to meet the load independently for a period of time which is called the autonomous hours.

3) Constraints of power and energy balance.

In the planning stage, we assume that the electricity stored in the batteries could maintain the average load demand for $\lambda$ hours:

$\lambda \cdot \overline{P_{L}^{y}} / \eta_{\text {inv }} \leq \eta_{\text {dis }} N_{o p, S_{i}}^{y} \cdot P_{d i s, \max }^{S_{i}}$

Together with the volume limit of the control room, the number of operating batteries has the following constraint:

$B_{S_{i}}^{y} \frac{\lambda \cdot \overline{P_{L}^{y}}}{\eta_{\text {inv }} \eta_{\text {dis }} P_{\text {dis, } \max }^{S_{i}}} \leq N_{o p, S_{i}}^{y} \leq B_{S_{i}}^{y} \frac{\text { Volarea }_{\text {alo }}}{\text { Vol }_{S_{i}}}$

where $\overline{P_{L}^{y}}$ is the average load demand, $P_{\text {dis,max }}^{S_{i}}$ is the maximum discharge power of the $S_{i}$ type batteries. 
As a crucial measure for load following, BESS has to manage power fluctuations caused by load and RES. Batteries should meet the instant power shortage between the peak load and the average power output of distributed energy resource. In the investment-decision model, the instant power balance requirements during the year are approximated by the following constraint:

$$
\begin{aligned}
& \eta_{i n v} \eta_{d i s} P_{m a x}^{S_{i}} N_{o p, S_{i}}^{y} \geq\left(1+r_{B}\right)\left(\widehat{P_{L}^{y}}-\overline{P_{w t}^{y}}-\overline{P_{p v}^{y}}-\overline{P_{d g}^{y}}\right) \\
& \overline{P_{w t}^{y}}=N_{w t}^{y} p_{w t, n} \cdot F L H_{w t}^{y} / 8760 \\
& \overline{P_{p v}^{y}}=N_{p v}^{y} p_{p v, n} \cdot F L H_{p v}^{y} / 8760 \\
& \overline{P_{d g}^{y}}=N_{d g}^{y} p_{d g, n} \cdot F L H_{d g}^{y} / 8760
\end{aligned}
$$

Where $P_{\max }^{S_{i}}$ is the maximum discharge power output. $r_{B}$ is the security coefficient introduced to cope with the uncertainty of renewable power and load. In this paper, the indicator full load hour (FLH) is defined as the ratio of annual energy production of each DER unit to its installed capacity. $F L H_{w t}^{y}, F L H_{p v}^{y}$ and $F L H_{d g}^{y}$ denote the FLHs of wind turbines, $\mathrm{PV}$ panels and diesel generators respectively.

\subsection{Microgrid long-term probabilistic sequential simulation}

In the life cycle planning of BESS, some key indicators need to be evaluated such as LPSP and yearly cycled energy of batteries. Therefore, the sequential Monte Carlo simulation is employed to deal with the problem [28].

In Monte Carlo simulation, the two-state Markov model is utilized to characterize the random contingency of each component in microgrid. Then, considering the intermittency and time-dependent feature of renewable energy generation, the output sequences of these components are sampled according to the time series models. To simplify the sample extraction process, normalized longterm hourly scenario database has been generated in advance, which speeds up the calculation of Monte-Carlo iterations.

After the sampling process, a heuristic energy management strategy is applied to simulate the detailed operation of the microgrid. The off-grid wind-solar-diesel microgrid should make full use of renewable energy to compensate the load demand. BESS and diesel generators are employed to cover the balanced load. The equations are as follows:

$\Delta P_{L}^{y}(t)=P_{L}^{y}(t)-N_{w t}^{y} \cdot p_{w t}^{y}(t)-N_{p v}^{y} \cdot p_{p v}^{y}(t)$

The operating constraints of batteries are shown as follows:

$\operatorname{SOC}_{S_{i}}^{\min } \leq \operatorname{SOC}_{S_{i}}(t) \leq \operatorname{SOC}_{S_{i}}^{\max }$

$0<P_{\text {dis, }, S_{i}}(t) \leq P_{\text {dis,max }}^{S_{i}}$

$0<P_{c h, S_{i}}(t) \leq P_{c h, \max }^{S_{i}}$

where $P_{c h, \text { max }}^{S_{i}}, P_{d i s, \text { max }}^{S_{i}}$ are the maximum charge and discharge rate of the type $S_{i}$ batteries.

If $\Delta P_{L}^{y}(t)<0$, it means the renewable energy supplied exceeds the need of demand. The redundant energy will be stored into batteries. If the batteries are fully charged, the spare energy will be wasted.

If $\Delta P_{L}^{y}(t)>0$, it means the load has not yet been met. The storage batteries will be discharged. If SOC gets below its lower limit, the batteries stop discharging. If the load is not satisfied, diesel generators will start. Regardless of the impact of ramp rate, the diesel generator will generate $\Delta P_{d g}^{y}(t)$ at hour $t$ :

$$
\Delta P_{d g}^{y}(t)= \begin{cases}0 & \Delta P_{L}^{y}(t)<P_{d i s, \text { max }}^{S_{i}} \\ \Delta P_{L}^{y}(t)-P_{d i s, \text { max }}^{S_{i}} & \Delta P_{L}^{y}(t)-P_{d i s, \text { max }}^{S_{i}}<P_{d g, \text { max }}^{y} \\ P_{d g, \text { max }}^{y} & \Delta P_{L}^{y}(t)-P_{d i s, \text { max }}^{S_{i}} \geq P_{d g, \text { max }}^{y}\end{cases}
$$

\section{Solution approach}

For microgrid in remote area or island, the life cycle planning of BESS is quite crucial. The long-term practical operation factors such as load growth, battery capacity fade and component random contingency have to be taken into account in planning stage. Thus the programming problem becomes a nonconvex nonlinear problem, which is difficult to be solved by analytical optimization algorithm. The decomposition-coordination approach is utilized to separate the problem into a main problem and long-term simulation constraints. Indicators that reflect the simulation result are continuously substituted to the main problem to change the solution space until an optimal result is acquired.

\subsection{The feedback mechanism and convergence criteria based on key indicators}

The key feedback variables of decompositioncoordination algorithm are the yearly full load hours (FLHs) of WT, PV and DG. FLHs of WT, PV and DG can be derived from microgrid long-term simulation. In the investment-decision model, FLHs has been substituted to constraint equation (26). If the relative error of FLHs with respect to that of previous iteration declines to a value small enough, it means the iteration process converges to a final state. A convergence index $D E_{i t r}$ can be expressed as

$D E_{i t r}=\frac{\sum_{i=\mathrm{WT}, \mathrm{PV}, \mathrm{DG}} \sum_{y=1}^{Y_{S}}\left(\left|\left(F L H_{i}^{y, t r}-F L H_{i}^{y, t t r-1}\right) p_{i, n} N_{i}^{y}\right|\right)}{\sum_{y=1}^{Y_{S}} \sum_{t=1}^{T} P_{L}^{y}(t)}$

where $F L H_{i}^{y, i t r}$ is the $y$ th year full load hour of a kind of DER in the itrth iteration. $p_{i, n}$ is the rated power of one DER unit. $N_{i}^{y}$ is the installed number of DER. If the convergence index $D E_{i t r}$ for the itrth iteration is smaller than a small enough value $\varepsilon$, the process stops. Otherwise it will run for $i t r_{\text {max }}$ times at most and stop.

LPSP is an index to characterize the reliability of a power system. State of Health $(\mathrm{SOH})$ indicates the remaining available capacity of batteries with respect to its nominal one. After carrying out the simulation, LPSP and SOH are evaluated to verify the feasibility of the planning.

LPSP is affected by the controllable components like the number of batteries and the reserve ratio of DG. LPSP in the $y$ th year should be less than an upper limitation. 


$$
\operatorname{LPSP}_{y}\left(N_{o p, S_{i}}^{y}, r_{d g}\right)<L P S P_{\max }
$$

$\mathrm{SOH}$ is a function of the number of batteries and the security coefficient of BESS. If the SOH of BESS is lower than $80 \%$, it ought to be replaced immediately. Therefore, the quantity of batteries should be sufficient to maintain the necessary $\mathrm{SOH}$.

$\mathrm{SOH}_{y}\left(N_{o p, S_{i}}^{y}, r_{B}\right)>80 \%$

\subsection{Decomposition-coordination algorithm}

The flow diagram for life cycle planning of BESS in an off-grid wind-solar-diesel microgrid is shown in Fig.3. The implementation is described according to the steps as follows:

Step 1: Initialize the number of iteration. Specify the location and configuration of the microgrid. Collect the historical data of renewable resources and the load demand. Specify the technical parameters of microgrid components.

Step 2: Determine the capacity of WT and PV for each planning year.

Step 3: Derive the capacity of diesel generators for each planning year.

Step 4: Save the life cycle configuration of DER as the input variable of the BESS planning. Initialize the key feedback variables that are the yearly FLHs of WT, PV and DG.

Step5: Update the number of iterations. Select the optimal battery type and calculate the number of batteries in the project lifespan according to the investment-decision objective function and constraints.

Step 6: Carry out the long-term microgrid simulation. Battery capacity loss is updated along with the charging/discharging cycles. If the batteries are replaced, the capacity loss is reset.

Step 7: Calculate the economic, reliability and operation performance indicators of the microgrid.

Step 8: Check whether the number of iterations reaches its maximum number.

Step 9: If the convergence criteria is not yet satisfied, update the FLHs of DER units and feed back to the investmentdecision model in step 5 .

Step 10: If the reliability requirement is not satisfied, the operating reserve ratio of diesel generators $r_{D G}$ needs to be adjusted by a fixed step value. Then the new coefficients will be substituted and the process will be restarted from step 2.

Step 11: If the $\mathrm{SOH}$ of batteries is not satisfied, the coefficient $r_{B}$ needs to be adjusted by a fixed step value. Then the new coefficients will be substituted and the process will be restarted from step 2 .

Remark 1: Note that each step in the proposed decomposition-coordination algorithm will be finished in a deterministic way. As long as the input parameters (including the scenarios for long-term simulation) are fixed, it follows a well-defined procedure (as in Steps 1-11) to deterministically generate and update the planning alternatives, which guarantees the uniqueness of the final solution. Moreover, the convergence condition (as defined in Section 4.1) provides an evaluation criterion to steadily yield a high-quality suboptimal solution of the original planning formulation.

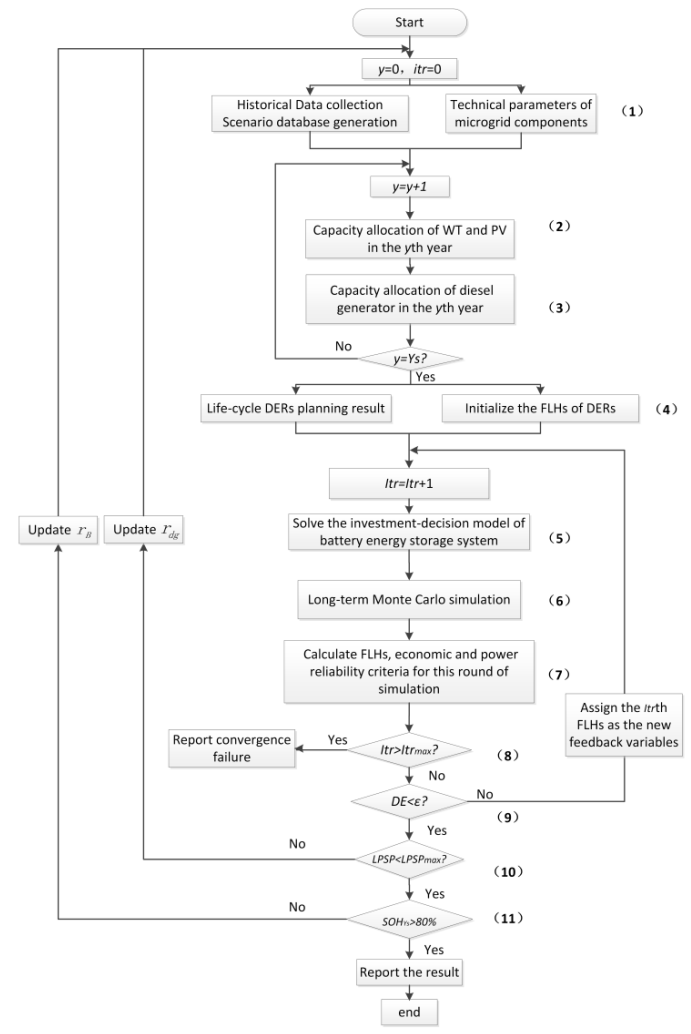

Fig.3. Main flowchart of the life time planning of battery energy storage system

\section{Case Studies}

Kythnos is one of the islands in the middle of the Aegean Sea in Greece. Kythnos microgrid is one of the pilot microgrids built by European MICROGRIDS project. In paper [29] the actual microgrid is presented. The microgrid is isolated from the distributed network of the island. A $20 \mathrm{~m}^{2}$ control room was built in the middle of the site to house battery inverters and its banks, diesel generators and its tank, and communication equipment. According to the MICROGRIDS project, the microgrid is composed of two subsystems. The first subsystem contains a $10 \mathrm{~kW}$ distributed photovoltaic systems with a $53 \mathrm{kWh}$ battery bank and a diesel generator with a nominal output of $5 \mathrm{kVA}$. The second one has $2 \mathrm{~kW}$ of PV panels mounted on the roof of the control room and a $32 \mathrm{kWh}$ battery bank. The second system is in a control room with $2 \mathrm{~kW}$ of PV panels mounted on the roof and a $32 \mathrm{kWh}$ battery bank inside. It provides electricity for the monitoring and communication needs.

In this section, we employed the proposed methodology considering the same conditions. Three types of tests are presented: Firstly, the complete methodology is applied to the Kythnos Island. Secondly an error analysis is 
illustrated. Thirdly a comparison with HOMER is presented assuming the load growth factor of the microgrid is zero.

The lifetime of the microgrid is 20 years, with a load growth of $2 \%$ each year. According to the longitude and latitude of Kythnos Island, the renewable resource data can be found from NASA Surface meteorology and Solar Energy Website [23]. The monthly average wind speed, solar radiation and temperature are listed in Table 1.

Table 1 Renewable resource data in Kythnos

\begin{tabular}{ccccc}
\hline $\begin{array}{c}\text { Mo } \\
\text { nth }\end{array}$ & $\begin{array}{c}\text { Clearness } \\
\text { index }\end{array}$ & $\begin{array}{c}\text { Monthly } \\
\text { Radiation } \\
\left(\mathrm{kWh} / \mathrm{m}^{2} / \text { day }\right)\end{array}$ & $\begin{array}{c}\text { Monthly Wind } \\
\text { Speed at 50m } \\
(\mathrm{m} / \mathrm{s})\end{array}$ & $\begin{array}{c}\text { Tempera } \\
\text { ture } \\
\left({ }^{\circ} \quad \mathrm{C}\right)\end{array}$ \\
\hline 1 & 0.473 & 2.213 & 7.230 & 11.0 \\
2 & 0.464 & 2.801 & 7.610 & 10.7 \\
3 & 0.531 & 4.212 & 6.460 & 12.4 \\
4 & 0.552 & 5.411 & 5.650 & 15.7 \\
5 & 0.574 & 6.350 & 5.050 & 20.0 \\
6 & 0.648 & 7.503 & 4.810 & 24.0 \\
7 & 0.647 & 7.303 & 6.120 & 25.9 \\
8 & 0.637 & 6.529 & 6.070 & 25.9 \\
9 & 0.637 & 5.453 & 5.470 & 23.3 \\
10 & 0.539 & 3.555 & 5.930 & 29.6 \\
11 & 0.472 & 2.347 & 6.500 & 15.4 \\
12 & 0.453 & 1.931 & 7.000 & 12.2 \\
\hline
\end{tabular}

The main electricity load is residential and commercial load. This paper uses a typical commercial and residential load curve [30] as the load curve. The annual peak load is $11.5 \mathrm{~kW}$.

In this paper, the microgrid is electrified mainly by RES such as wind turbine and photovoltaic arrays. BESS and diesel generators are used to coordinate with renewable power generators. In this paper, the microgrid is electrified mainly by RES such as wind turbine and photovoltaic arrays. BESS and diesel generators are used to coordinate with renewable power generators. Table 2 lists the specification of wind turbine, PV panel and diesel generator. Table 3 provides data of optional storage batteries.

According to Table 2, Fig.4 depicts the weekly PV power output of a $0.2 \mathrm{~kW} \mathrm{PV}$ panel, WT power output of a $2 \mathrm{~kW}$ wind turbine and hourly load curve for the first year.

\subsection{Life-cycle planning of BESS}

Fig. 5 shows the allocated capacities of wind turbines, PV panels and diesel generators. As the load demand increases, the number of wind turbines increases slightly while the number of PV panels increases in a more sensitive way. This is because the panels have smaller rated capacity and can be dispersedly mounted above household roofs.

The average relative variation between load and renewable power $D_{L}$ for 20 years is 1.39 . According to the allocation result of WT, PV and DG, the battery energy storage system will be optimally sized. Among the three types of batteries, the lead-acid batteries are finally chosen Assuming the lifetime of lead-acid batteries are 5 years, they will be replaced for 4 times. The loss of battery capacity along the years is shown in Fig.6. The total capacity of BESS increases periodically as load demand grows. Moreover, the available capacity will continue to fade along with the battery charging/discharging cycles. If the loss of capacity was not considered, the accuracy of optimal configuration results might be affected. Microgrid energy production of different sources is shown in Fig.7. It can be seen that, with the help of BESS, renewable energy offers $80 \%$ of the total energy consumption.

Fig. 8 shows the annual cash flow in the considered planning period. Initial investment accounts for the largest part and fuel cost is relatively high (white bar). The cost increment in the twelfth year is mainly caused by the growing number of wind turbines. Replaced with new storage batteries, the replacement fee is showed as the grey bar respectively in years 6,11 and 16 .

Table 2 Parameters of distributed generators

\begin{tabular}{ccc}
\hline Photovoltaic Panel & Wind Turbine Generator & ECO2kW \\
BP-solar 3200 & Price: $3000 €$ & Price: $100 € / \mathrm{kW}$ \\
Price: $390 € /$ panel & O\&M cost: $6 € /$ year & O\&M cost: $0.004 € / \mathrm{hr}$ \\
O\&M cost: $0.6 € /$ year & Lifetime: 20 years & Lifetime: $20000 \mathrm{hrs}$ \\
Lifetime: 20 years & Rated power: $2 \mathrm{~kW}$ & Diesel price: $1.3 € / \mathrm{L}$ \\
Rated power: $0.2 \mathrm{~kW}$ & Cut-in speed: $3 \mathrm{~m} / \mathrm{s}$ & Fuel consumption a: $.01 \mathrm{~L} / \mathrm{hr} / \mathrm{kW}(\mathrm{rate})$ \\
Open-circuit voltage: $30.8 \mathrm{~V}$ & Cut-out speed: $24 \mathrm{~m} / \mathrm{s}$ & Fuel consumption b: $0.29 \mathrm{~L} /(\mathrm{kWh})$ \\
Short-circuit current: $8.7 \mathrm{~A}$ & Rated speed: $8 \mathrm{~m} / \mathrm{s}$ & -- \\
Efficiency: $18 \%$ & Blade length: $2 \mathrm{~m}$ & - \\
Area: $1.4 \mathrm{~m}^{2}$ & Hub height: $8 \mathrm{~m}$ & - \\
Operation current: $47{ }^{\circ} \mathrm{C}$ & MTTF: $950 \mathrm{hr}$ & MTTF: $950 \mathrm{hr}$ \\
MTTF: $950 \mathrm{hr}$ & MTTR: $50 \mathrm{hr}$ & MTTR: $50 \mathrm{hr}$ \\
MTTR: $50 \mathrm{hr}$ & &
\end{tabular}


Table 3 Parameters of optional storage battery

\begin{tabular}{|c|c|c|c|c|c|c|}
\hline Battery Type & $\begin{array}{l}\text { Rated Capacity } \\
\text { (Ah) }\end{array}$ & $\begin{array}{l}\text { Rated Voltage } \\
\text { (V) }\end{array}$ & $\begin{array}{c}\text { Initial cost } \\
(€)\end{array}$ & $\begin{array}{c}\text { O\&M cost } \\
(€)\end{array}$ & $\begin{array}{c}\text { Recovery cost } \\
(€)\end{array}$ & $\begin{array}{l}\text { Volume } \\
\left(\mathrm{m}^{3}\right)\end{array}$ \\
\hline Lead-acid & 83.4 & 12 & 86 & 2 & -0.5 & 0.013 \\
\hline $\mathrm{NaS}$ & 650 & 2 & 260 & 4 & 5 & 0.010 \\
\hline Li-ion & 200 & 3.3 & 215 & 2 & 0.1 & 0.004 \\
\hline Battery Type & $\begin{array}{l}\text { Lifetime } \\
\text { (year) }\end{array}$ & $\begin{array}{c}\text { Max DOD } \\
(\%) \\
\end{array}$ & Efficiency & $\begin{array}{l}\text { Max charge } \\
\text { current }(\mathrm{A})\end{array}$ & $\begin{array}{l}\text { Max discharge } \\
\text { current }(\mathrm{A})\end{array}$ & $\begin{array}{c}\text { Cycles to failure } \\
\text { (Times) }\end{array}$ \\
\hline Lead-acid & 5 & 70 & 0.80 & 83.4 & 83.4 & 818 \\
\hline $\mathrm{NaS}$ & 9 & 90 & 0.85 & 325 & 650 & 4000 \\
\hline Li-ion & 8 & 80 & 0.93 & 100 & 200 & 3000 \\
\hline
\end{tabular}

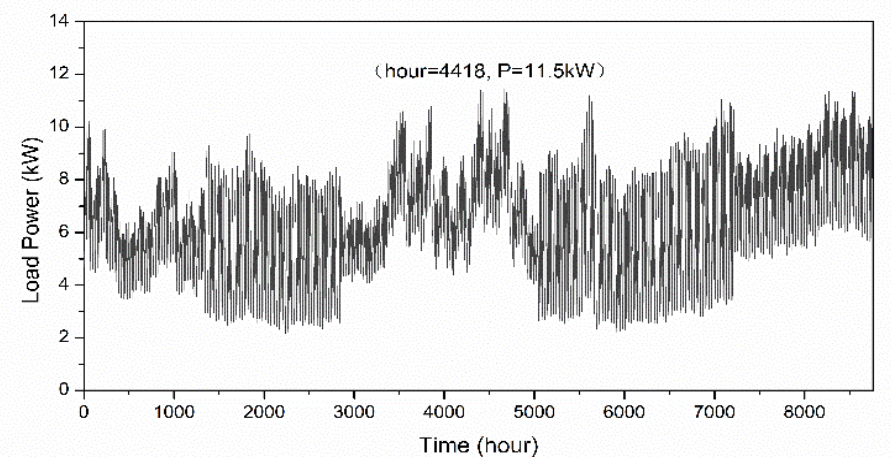

Fig.4. Historical data of renewable sources and the load

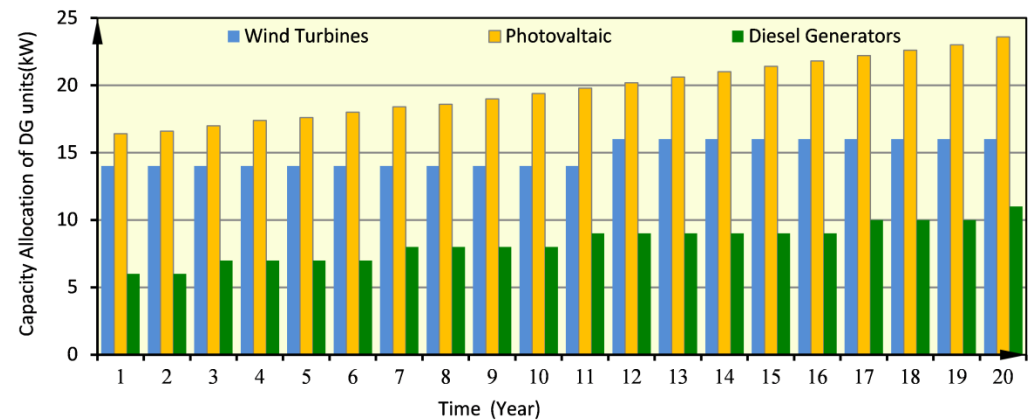

Fig. 5. Capacity allocation results of DER for 20 years

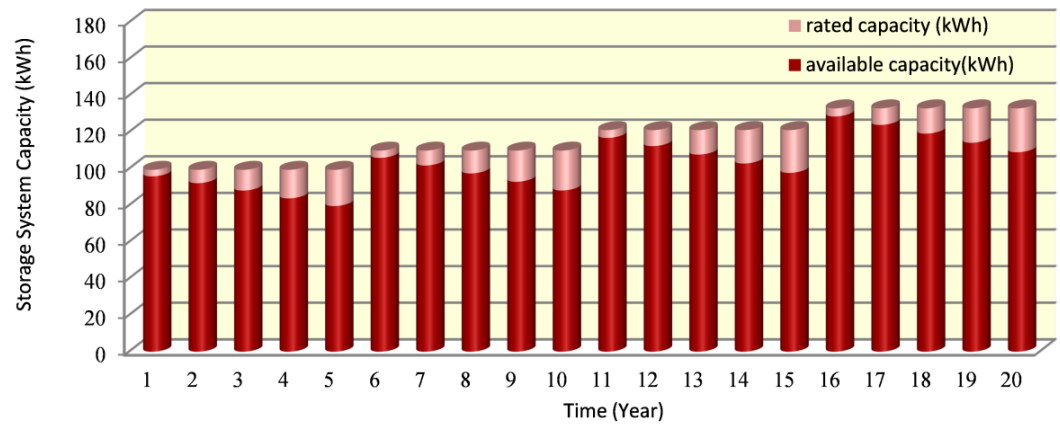

Fig.6. The comparison of battery rated capacity and available capacity along the years 


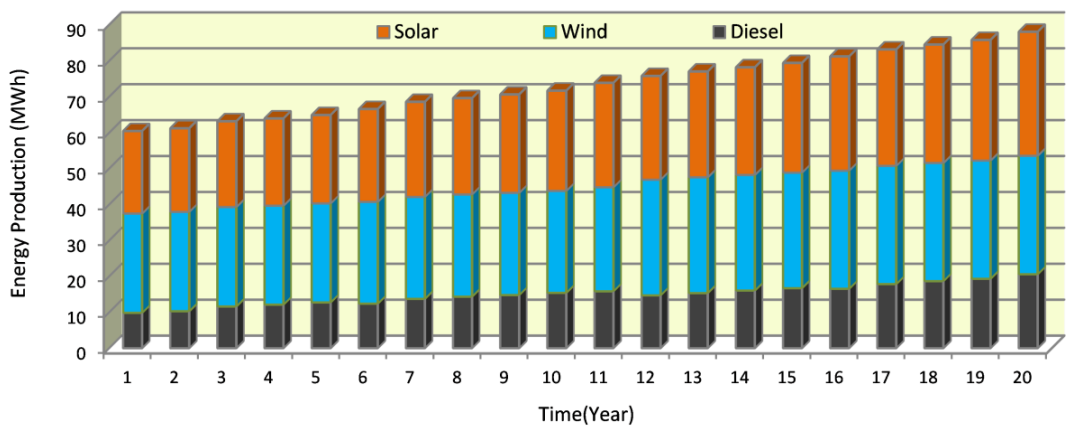

Fig.7. Microgrid energy production of different sources

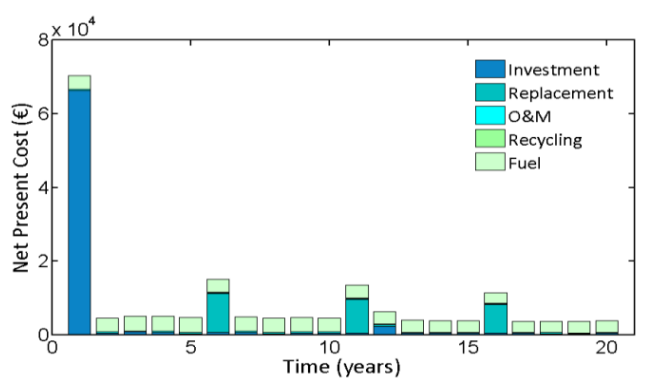

Fig.8. The annual cash flow for the microgrid in planning period

The main economic indicators, net present cost is $180,149 €$, and levelized cost of energy is 21.4 cents/kWh. If the system only allocates $17 \mathrm{~kW}$ diesel generators to meet the load, the net present cost will be no less than $355,522 €$, of which the fuel costs have far exceeded the total cost of proposed project. The diesel-powered system will have the levelized cost up to 40 cents $/ \mathrm{kWh}$. The literature [31] reported that the LCE of Kythnos Island Microgrid is about 30 cents $/ \mathrm{kWh}$. Therefore, the proposed sizing result is more economical than diesel-electrified system and the practical one.

As mentioned above, the energy production of DER can be derived by multiplying the FLH by its rated power. Fig.7 illustrates the energy production of each DER in 20 years. Cooperating with BESS, wind and solar energy production account for respectively $41 \%, 39 \%$ of the total energy production and the fuel-consumed energy takes the rest $20 \%$ for 20 years.

To illustrate the properties of the proposed method, one representative week is selected to illustrate the simulation and operation of various components in microgrid under different circumstances. Fig.9 (a) shows that the power output of photovoltaic panels and wind turbines contributes to a certain extent to the load. Fig.9 (b) shows the SOC of energy storage batteries and diesel generator power output. Battery energy storage system allows the full use of renewable power sources. Diesel generators can provide sufficient backup if necessary.

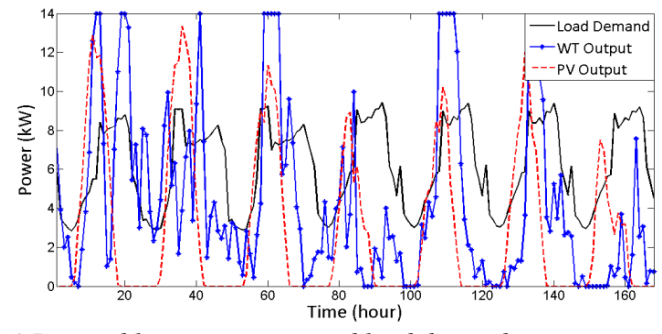

(a) Renewable power output and load demand

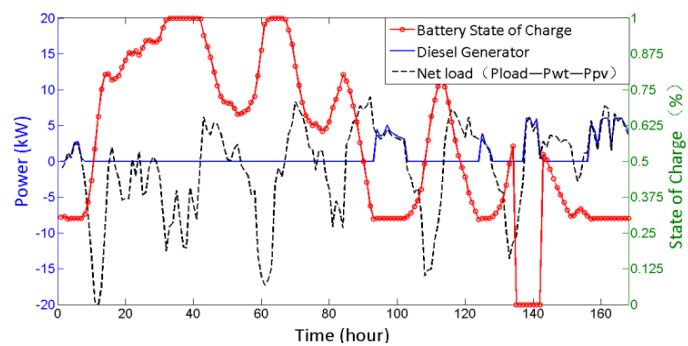

(b) Battery charging/discharging power and diesel generator operation

Fig.9. The coordination operation of components in the microgrid

Overall, a practical microgrid operation simulation can be divided into the following situations:

(1) During the first 3 days (hour 1-72), renewable DER units have large power output. The net load is always less than 0 so that the energy storage batteries are usually charged and only release a certain amount of energy at night. Diesel generators are not used.

(2) During the next 2 days (hour 73-121), renewable DER units have less power output. The energy storage batteries have insufficient capacity to sustain the demand. So, the SOC is kept at $30 \%$, i.e., the lower limit. The diesel generator needs to be started to supply the load.

(3) In the $6^{\text {th }}$ day (hour 122-146), a failure occurs to the battery energy storage system. The battery will neither charge nor discharge. The diesel generators have to generate power to meet the remaining load as much as possible.

(4) In the $7^{\text {th }}$ day, the battery energy storage system is recovered. Since the power output of renewable sources is 
small, the storage batteries keep discharge and coordinate with diesel generators to meet the load.

\subsection{Error analysis}

The decomposition-coordination method needs a few iterations to find the final result. In this study, the convergence index $D E_{i t r}$ demonstrates the process of coordination iteration.

Before simulation, the annual electricity supply and the full load hours of renewable distributed generators are unknown. They are related to the abundance of renewable resource and the available amount of energy that could be stored in batteries. Hence, the loss of load and operation cost of microgrid cannot be defined before simulation.

Diesel generator, for example, is here to show the iteration process and full load hour convergence in Table 4. Firstly, initial FLH, 2000 hours, is assumed into investmentdecision model. Then the microgrid is simulated for 20 years. New FLH of diesel generator is achieved and fed back to equations (25), (26) and (27) to refresh the result of battery storage system.

Table 5 shows that the variation of FLHs is smaller than 0.003 after 5 iterations.

\subsection{Comparison with HOMER results}

HOMER software is hybrid power system analysis and design software developed by the US National Renewable Energy Experiments (NREL) in 1993. This software has been widely recognized and applied to hybrid power system techno-economic analysis. In this paper, a comparative analysis will be presented for the results of the proposed method and HOMER.

HOMER will enumerate all the possible values to find several feasible solutions that meet the microgrid operation requirements, and sort them according to the net present cost. To facilitate the analysis, only load following mode (LF mode) is used as the control mode.

Table 4 The iteration process and diesel generator full load hour convergence

\begin{tabular}{|c|c|c|c|c|c|c|}
\hline \multirow{2}{*}{ Year } & \multicolumn{6}{|c|}{ Diesel Generator FLH } \\
\hline & Itr 0 & Itr 1 & $\operatorname{Itr} 2$ & Itr 3 & Itr 4 & Itr 5 \\
\hline 1 & 2000 & 1589 & 1640 & 1654 & 1640 & 1642 \\
\hline 2 & 2000 & 1722 & 1724 & 1721 & 1724 & 1734 \\
\hline 3 & 2000 & 1616 & 1663 & 1670 & 1638 & 1667 \\
\hline 4 & 2000 & 1708 & 1721 & 1729 & 1747 & 1731 \\
\hline 5 & 2000 & 1805 & 1834 & 1811 & 1848 & 1819 \\
\hline 6 & 2000 & 1733 & 1766 & 1760 & 1791 & 1769 \\
\hline 7 & 2000 & 1662 & 1707 & 1696 & 1686 & 1716 \\
\hline 8 & 2000 & 1765 & 1814 & 1775 & 1791 & 1792 \\
\hline 9 & 2000 & 1806 & 1855 & 1862 & 1869 & 1847 \\
\hline 10 & 2000 & 1898 & 1942 & 1927 & 1926 & 1925 \\
\hline 11 & 2000 & 1761 & 1775 & 1760 & 1763 & 1759 \\
\hline 12 & 2000 & 1579 & 1625 & 1637 & 1632 & 1633 \\
\hline 13 & 2000 & 1677 & 1706 & 1725 & 1697 & 1703 \\
\hline 14 & 2000 & 1736 & 1790 & 1784 & 1770 & 1782 \\
\hline 15 & 2000 & 1815 & 1870 & 1861 & 1860 & 1855 \\
\hline 16 & 2000 & 1805 & 1825 & 1845 & 1834 & 1835 \\
\hline 17 & 2000 & 1760 & 1786 & 1793 & 1796 & 1783 \\
\hline 18 & 2000 & 1825 & 1867 & 1854 & 1854 & 1865 \\
\hline 19 & 2000 & 1917 & 1936 & 1931 & 1953 & 1931 \\
\hline 20 & 2000 & 1838 & 1884 & 1871 & 1907 & 1870 \\
\hline DE & --- & 0.805 & 0.01 & 0.003 & 0.003 & 0.003 \\
\hline $\operatorname{BSS}(€)$ & --- & 48675 & 43649 & 43843 & 43947 & 43793 \\
\hline $\operatorname{NPC}(€)$ & --- & 183736 & 180160 & 180226 & 180434 & 180149 \\
\hline
\end{tabular}

Since HOMER is not possible to take into account any load growth along the planning horizon, for comparison, the same case without load growth is solved by the proposed approach.

Fig.10 illustrates the rated power of distributed energy sources and available capacity of battery energy storage system obtained by the proposed method, HOMER and the practical configuration without load growth. It can be seen that the installed numbers of WT and PV panels of the proposed method are smaller than that of HOMER. However the battery bank capacity of the proposed method is larger than that of HOMER. It is because battery capacity fade is considered in this study while in HOMER this factor is neglected along the project lifespan.

In this paper, the comparison of economic and technique indicators of the proposed method and HOMER software is listed in Table 5. In general, the LPSP of both methods are comparable. And the batteries in two systems can both sustain for more than 5 years' lifetimes. However the net present cost and the annualized fuel cost of proposed method is greater than the costs of HOMER, respectively. It is because random failures of microgrid components are 
considered in this study while the reliability of microgrid components in HOMER is $100 \%$. Therefore at the comparable level of LPSP, the proposed planning scheme costs more to handle with the uncertainty of renewable energy sources, the primary load and component random failures.

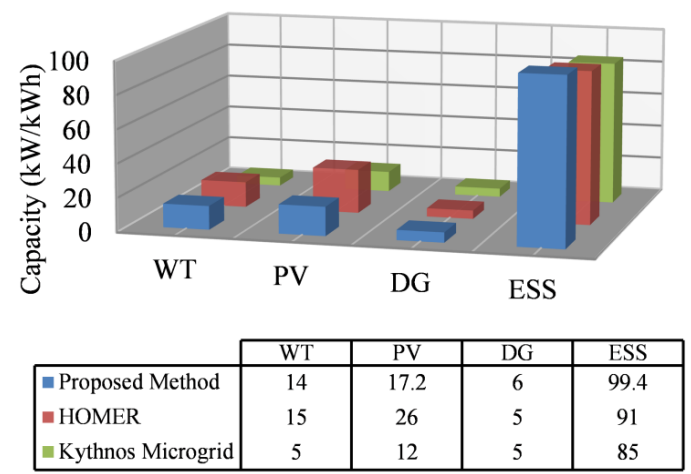

Fig.10. The different configurations of Kythnos Island obtained by different methods

Table 5 The comparison of proposed method and HOMER

\begin{tabular}{ccc}
\hline Economic and & Proposed & HOMER \\
technological indicator & Method & \\
\hline Net present cost $(€)$ & 141,623 & 118,437 \\
LCE $(€ / \mathrm{kWh})$ & 0.2046 & 0.1740 \\
Initial capital cost $(€)$ & 66814 & 75071 \\
Annual fuel cost $(€)$ & 3939 & 2242 \\
LPSP & 0.0277 & 0.0276 \\
EENS $(\mathrm{kWh} / \mathrm{yr})$ & 1539 & 1531 \\
Energy excess rate $(\%)$ & 0.1307 & 0.1468 \\
Battery throughput & 16197 & 14393 \\
(kWh/yr) & & \\
WTG Full load & 1924 & 1805 \\
hours(hr) & 1364 & 1667 \\
PV Full load hours $(\mathrm{hr})$ & 1659 & 1132 \\
DG Full load hours(hr) & 155 & 158 \\
Battery charge cycles & 155 \\
\hline
\end{tabular}

In conclusion, several advantages are possessed by the proposed method. Compared with HOMER, the proposed method can consider load growth factor, battery capacity fade and component random failures. At the perspective of operation, it can provide the life cycle planning of battery energy storage system and microgrid performance indicators for the planning period, which offers meaningful reference for microgrid sizing and electricity market price setting.

\section{Conclusions}

This paper puts forward a life cycle planning of battery energy storage system in an off-grid wind-solardiesel microgrid where the dynamic factors such as demand growth, battery capacity fading, and components' contingencies are well-considered under a multi-stage and multi-timescale decision framework. In the first stage, Multi-timescale BESS modelling is established. The optima DER capacity allocation is carried out to meet load demand. While in the second stage, the investment decisions of BESS are made periodically to yield the type selection and life cycle plans of BESS. And the long-term probabilistic sequential simulation is adopted to comprehensively evaluate the investment decisions and derive detailed operation indicators. Decomposition-coordination algorithm is developed to address the presented planning model, which iteratively strengthens the feasible space of investment decision model by substituting the operation indicators until an acceptable sub-optimal solution is obtained. In the third stage, the performance of optimal configuration will be tested and evaluated. A case study of practical microgrid is demonstrated. Compared with HOMER's result, the total cost of the proposed method is essentially the same at a comparable level of reliability, which validates the feasibility and effectiveness of this method. This work can provide a more practical and precise BESS type selection, capacity determination and replacement plan during the overall microgrid lifetime which is of practical reference for the BESS planning in off-grid microgrids.

In future studies, more sophisticated battery model and flexible energy management strategies, which can refine the sizing results, will be developed and extended in our proposed optimization framework.

\section{Acknowledgement}

The work has been supported by National Natural Science Foundation of China (51777155); National Key R\&D Program of China (2018YFB0905000); Key Research and Development Program of Shaanxi (2017ZDCXL-GY02-03).

\section{References}

[1] Liu YF, Yu SS, Zhu Y, et al, Modeling, planning, application and management of energy systems for isolated areas: A review, Renewable and Sustainable Energy Reviews, 2018,82(1): 460-470.

[2] Razman A, Normazlina MI, Chee WT, Components sizing of photovoltaic stand-alone system based on loss of power supply probability, Renewable and Sustainable Energy Reviews, 2018,81(2): 2731-2743

[3] Imre G. Grid Energy Storage. USA: U.S. Department of Energy; 2013

[4] Saez-De-Ibarra A, Milo A, Gaztanaga H, et al. Co-Optimization of Storage System Sizing and Control Strategy for Intelligent Photovoltaic Power Plants Market Integration. IEEE Transactions on Sustainable Energy, 2016,7(4):1749-1761.

[5] Zidar M, Georgilakis PS, Hatziargyriou ND, et al. Review of energy storage allocation in poewr distribution networks: applications, methods and future research. IET Generation Transmission \& Distribution, 2016,10 (3):645-652.

[6] Lambert T, Gilman P, Lilienthal P. Micropower system modeling with homer. New York: John Wiley \& Sons, Inc.; 2006.

[7] Zhang YM, Tang XS, Qi ZP, et al. The Ragone plots guided sizing of hybrid storage system for taming the wind power. Internationa Journal of Electrical Power \& Energy Systems,2015,65(1):246-253.

[8] Wen SL, Lan H, Yu d, Fu Q, et al. Optimal sizing of hybrid energy storage sub-systems in PV/diesel ship power system using frequency analysis, Energy, 2017,140(1): 198-208.

[9] Jiang QY, Hong HS. Wavelet-based capacity configuration and coordinated control of hybrid energy storage system for smoothing out wind power fluctuations. IEEE Transactions on Powe Systems, 2013,28(2):1363-1372.

[10] Xiao J, Bai LQ, Li FX, et al. Sizing of energy storage and diese generators in an isolated microgrid using discrete fourier transform (DFT). IEEE Transactions on Sustainable Energy, 2014 5(3):907-916. 


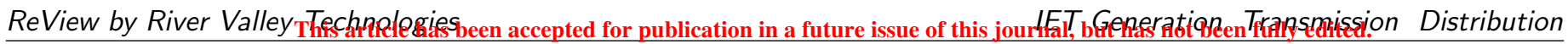
Content may change prior to final publication in an issue of the journal. To cite the paper please use the doi provided on the Digital Library page.

[11] Mallol-Poyato R, Jimnez-Fernndez S, Daz-Villar P, et al. Joint optimization of a microgrid's structure design and its operation using a two-steps evolutionary algorithm. Energy,2016,94:775-785.

[12] Ibrahim AI, Tamer K, Azah M. Impact of Battery's Model Accuracy on Size Optimization Process of a Standalone Photovoltaic System. Sustainability, 2016,8(9):894-906.

[13] Amrollahi M, Bathaee S. Techno-economic optimization of hybrid photovoltaic/wind generation together with energy storage system in a stand-alone micro-grid subjected to demand response. Applied Energy, 2017,202: 66-77.

[14] Paliwal P, Patidar NP, Nema RK. Determination of reliability constrained optimal resource mix for an autonomous hybrid power system using particle swarm optimization. Renewable Energy,2014,63(1):194-204.

[15] Xu L, Ruan XB, Mao CX, et al. An improved optimal sizing method for wind-solar-battery hybrid power system. IEEE Transactions on Sustainable Energy,2013,4(3):774-785.

[16] Arabali A, Ghofrani M, Etezadi-Amoli M, et al. Stochastic performance assessment and sizing for a hybrid power system of solar/wind/energy storage. IEEE Transactions on Sustainable Solar/wind/energy storage.

[17] Tamer K, Ibrahim AI, Azah M. A review on sizing methodologies of photovoltaic array and storage battery in a standalone photovoltaic system. Energy Conversion and Management,2016,120:430-448.

[18] Wang S, Li Z, Wu L, et al. New Metrics for Assessing the Reliability and Economics of Microgrids in Distribution System. IEEE Transactions on Power Systems, 2013,28(3): 2852-2861.

[19] Shang C, Dipti S, Thomas R. Generation-scheduling-coupled battery sizing of stand-alone hybrid power systems. Energy,2016,114:671682

[20] Cao XY, Wang JX, Zeng B. A chance constrained information-gap decision model for multi-period microgrid planning. IEEE Transactions on Power Systems, 2018,33(3): 2684-2695.

[21] Wang XF, McDonald J. Modern power system planning. McGrawHill Companies, 1994.

[22] Sperck R, Daniel K,Chris M. Issues in electricity planning with computer models: illustration with Elfin and WASP. Utilities Policy,1998,7(1):201-219.

[23] Elena MK, John C, Craig B. A comparison of lead-acid and lithiumbased battery behavior and capacity fade in off-grid renewable charging applications. Energy,2013, 60(1):492-500.

[24] Masoud MT, Mohammad-Reza HY, Vahid E, Ali Safaei. Optimum sizing and optimum energy management of a hybrid energy storage system for lithium battery life improvement. Journal of Power Sources, 2013,244(1):2-10.

[25] National Aeronautics and Space Administration (NASA). The Atmospheric Science Data Center (ASDC), 2014. Atmospheric science

[26] Zhang N, Kang CQ, Duan CG, et al. Simulation methodology of multiple wind farms operation considering wind speed correlation. International Journal of Electrical Power and Energy Systems, 2010,30(4):264-273

[27] Mora-López L, Sidrach-De-Cardona M. Multiplicative ARMA models to generate hourly series of global irradiation. Solar Energy, 1998, 63(5):283-291.

[28] Sankarakrishnan A, Billinton R. Sequential Monte Carlo simulation for composite power system reliability analysis with time varying loads. IEEE Transactions on Power Systems, 1995,10:1540-1545.

[29] The National Technical University (NTUA). Pilot Kythnos Microgrid,2010

http://www.microgrids.eu/index.php?page=kythnos\&id=2

[30] Jardini JA, Tahan C, Gouvea MR, et al. Daily load profiles for residential, commercial and industrial low voltage consumers. IEEE Transactions on Power Delivery,2002,15(1):375-380.

[31] Jayanta DM, Nikos K. Overview of challenges, prospects, environmental impacts and policies for renewable energy and sustainable development in Greece. Renewable and Sustainable Energy Reviews,2013,23(1):431-442. 